Original Research Paper

\title{
On Almost Periodic Solutions of Abstract Semilinear Fractional Inclusions with Weyl-Liouville Derivatives of Order $\gamma \in(\mathbf{0}, \mathbf{1}]$
}

\author{
Marko Kostić \\ Department of Fundamental Sciences, Faculty of Technical Sciences, University of Novi Sad, Serbia
}

\section{Article history}

Received: 18-05-2017

Revised: 03-06-2017

Accepted: 25-07-2017

Email: marco.s@verat.net

\begin{abstract}
The main aim of this paper is to examine the existence and uniqueness of almost periodic solutions for a class of (semilinear) fractional relaxation inclusions with Stepanov almost periodic coefficients. We deal with the Weyl-Liouville fractional derivatives of order $\gamma \in(0,1]$, paying special attention to the analysis of semilinear differential inclusions of first order. We use the results from the theory of fractional powers of sectorial multivalued linear operators to achieve our goals, providing an interesting application to semilinear fractional Poisson heat equation in $L^{p}$-spaces.
\end{abstract}

Keywords: Weyl-Liouville Fractional Derivatives, Almost Periodicity, Stepanov Almost Periodicity, Multivalued Linear Operators, Fractional Powers of Operators, 2010 Mathematics Subject Classification, Primary 34A60, 47D06; Secondary 47D03, 47D99

\section{Introduction and Preliminaries}

In (Section 6.3), Pazy (1983) has analyzed semilinear Cauchy problems with generators of analytic strongly continuous semigroups by using the results from the theory of fractional powers of operators. Concerning the study of existence and uniqueness of almost periodic solutions of non-degenerate semilinear Cauchy problems, it seems that the fractional powers of operators has been employed for the first time by Bahaj and Sidki (2002) (cf. also Diagana et al. (2006) and El-Borai and Debbouche (2009)).

Suppose that $\gamma \in(0,1]$ and $A$ is a multivalued linear operator on a Banach space $X$ satisfying the condition (Favini and Yagi, 1998, (P), p. 47) examined by Favini, Yagi:

(P) There exist finite constants $c, M>0$ and $\beta \in(0$, 1] such that:

$$
\Psi:=\Psi_{c}:=\{\lambda \in \mathbb{C}: \Re \lambda \geq-c(|\Im \lambda|+1)\} \subseteq \rho(A)
$$

and:

$$
\|R(\lambda: A)\| \leq M(1+|\lambda|)^{-\beta}, \lambda \in \Psi
$$

Of concern is the following abstract Cauchy inclusion of first order: $u^{\prime}(t) \in A u(t)+f(t, u(t)), \quad t \in \mathbb{R}$

and its fractional relaxation analogue:

$D_{t,+}^{\gamma} u(t) \in A u(t)+f(t, u(t)), \quad t \in \mathbb{R}$

where, $D_{t,+}^{\gamma}$ denotes the Weyl-Liouville fractional derivative of order $\gamma, x_{0} \in X$ and $f: \mathbb{R} \times X \rightarrow X$ is Stepanov almost periodic.

In the present paper, we use fractional powers of sectorial multivalued linear operators with a view of establishing new structural results on the existence and uniqueness of almost periodic solutions of semilinear Cauchy inclusions (1.1) and (1.2). Our results seem to be new even for fractional relaxation equations with almost sectorial operators (Periago and Straub, 2002).

It is worth noting that the conditions on function $f(\cdot, \cdot)$ used in this study are weaker than those employed in (Bahaj and Sidki, 2002) for the Equation 1.1, resp., (El-Borai and Debbouche, 2009) for the Equation 1.2, where the authors have looked into the following condition (known already from (Pazy, 1983)):

The function $f: \mathbb{R} \times\left[D\left((-A)^{\emptyset}\right)\right] \rightarrow X$ satisfies that there are finite numbers $L>0$ and $\eta \in[0$, 
1] such that $\left\|f\left(t_{1}, x_{1}\right)-f\left(t_{2}, x_{2}\right)\right\| \leq L\left(\left|t_{1}-t_{2}\right|^{\eta}+\right.$ $\left.\left\|x_{1}-x_{2}\right\|_{\theta}\right)$ for all $\left(t_{1}, x_{1}\right),\left(t_{2}, x_{2}\right)$ in $\mathbb{R} \times$ $\left[D\left((-A)^{\circ}\right)\right] ;-A$ is the single-valued generator of an exponentially decaying $C_{0}$-semigroup.

In this case, the mild solutions constructed for (1.1) and (1.2) are Hölder continuous, which are no longer true in our approach (without going into further details, we want only to observe here that the structural results from (Bahaj and Sidki, 2002; El-Borai and Debbouche, 2009) can be reconsidered for degenerate semigroups of operators). It is clear that the condition (F) implies the validity of usually considered Lipschitz type condition (4.2) used below and that the validity of (4.2) or its generalization (4.1) used below does not imply the validity of condition $(\mathrm{F})$. Furthermore, we consider the case in which the function $f(\cdot, \cdot)$ is not uniformly almost periodic but only Stepanov almost periodic.

The organization of paper can be brifley described as follows. In section 2, we recall the basic facts about almost periodic functions and Stepanov almost periodic functions that will be necessary for our further work. The main aim of section 3 is to remind us of some elementary definitions and results from the theory of multivalued linear operators in Banach spaces; fractional powers and interpolation spaces of multivalued linear operators are examined in a separate subsection. Our main results and contributions are given in Section 4, which are divided into two separate subsections. In Subsection 4.1, we investigate the almost periodic solutions of abstract Cauchy inclusion (1.1), while in Subsection 4.2 we investigate the almost periodic solutions of abstract Cauchy inclusion (1.2).

We use the standard notation throughout the paper. By $\mathrm{X}$ and $\mathrm{Y}$ we denote two Banach spaces over the field of complex numbers. The symbol $L(\mathrm{X}, Y)$ stands for the space consisting of all continuous linear mappings from $X$ into $Y ; L(X) \equiv L(X, X)$ and $\|\cdot\|$ stands for the norm of $X$. Denote by $C_{b}(\mathbb{R}: X)$ the vector space consisted of all bounded continuous functions from $\mathbb{R}$ into $X$; the supnorm turns this space into one of Banach's. The Gamma function is denoted by $\Gamma(\cdot)$ and the principal branch is always used to take the powers. Define $g_{\alpha}(t):=$ $t^{\alpha-1} / \Gamma(\alpha), t>0(\alpha>0)$.

Fractional calculus and fractional differential equations have gained much attention recently due to their numerous applications (Bazhlekova, 2001; Diethelm, 2010; Kostić, 2011; 2015; Prüss, 1993; Samko et al., 1993; von Wahl, 1972). We need to recall some basic facts about the Wright functions, which are known to play an important role in fractional calculus. Let $\gamma \in(0,1)$. Then the Wright function $\Phi_{\gamma}(\cdot)$ is defined by the formula:

$$
\Phi_{\gamma}(z):=\sum_{n=0}^{\infty} \frac{(-z)^{n}}{n ! \Gamma(1-\gamma-\gamma n)}, z \in \mathbb{C}
$$

Let us recall that $\Phi_{\gamma}(\cdot)$ is an $R$ entire function, as well as that $\Phi_{\gamma}(t) \geq 0, t \geq 0, \int_{0}^{\infty} t^{r} \Phi_{\gamma}(t) d t=\frac{\Gamma(1+r)}{\Gamma(1+\gamma r)}, r>-1$ and $\int_{0}^{\infty} e^{-z t} \Phi_{\gamma}(t) d t=E_{\gamma}(-z), \quad z \in \mathbb{C}$, where $E_{\gamma}(\cdot)$ denotes the Mittag-Leffer function. For further information concerning the Mittag-Leffer and Wright functions, the reader may consult the doctoral dissertation of Bazhlekova (2001) and references cited therein.

The Weyl-Liouville fractional derivative $D_{t,+}^{\gamma} u(t)$ of order $\gamma$ is defined for those continuous functions $u: \mathbb{R} \rightarrow$ $X$ such that $t \mapsto \int_{-\infty}^{t} g_{1-\gamma}(t-s) u(s) d s, \quad t \in \mathbb{R}$ is a welldefined continuously differentiable mapping, by:

$$
D_{t,+}^{\gamma} u(t):=\frac{d}{d t} \int_{-\infty}^{t} g_{1-\gamma}(t-s) u(s) d s, t \in \mathbb{R}
$$

Set $D_{t,+}^{\gamma} u(t):=-(d / d t) u(t)$. For more details about the Weyl-Liouville fractional derivatives, we refer the reader to the paper by Mu et al. (2017).

\section{Almost Periodic Functions and Stepanov Almost Periodic Functions}

It is well known that the class of almost periodic function was introduced by Bohr in 1925 and later generalized by many other mathematicians (for further information, the reader may consult the monographs (Diagana, 2013; N'Guérékata, 2001; Hino et al., 2002; Levitan and Zhikov, 1982)). Let $I=\mathbb{R}$ or $I=[0, \infty)$ and let $f: I \rightarrow X$ be continuous and let $\epsilon>0$. Then we say that a number $\tau>0$ is an $\epsilon$-period for $f(\cdot)$ iff $\|f(t+\tau)-f(t)\| \leq \epsilon$, $t \in I$. The set constituted of all $\epsilon$-periods for $f(\cdot)$ is denoted by $\vartheta(f, \epsilon)$. We say that $f(\cdot)$ is almost periodic, a.p. for short, iff for each $\epsilon>0$ the set $\vartheta(f, \epsilon)$ is relatively dense in $I$, which means that there exists $l>0$ such that any subinterval of $I$ of length $l$ meets $\vartheta(f, \epsilon)$. The space of all almost periodic functions from the interval $I$ into $X$ will be denoted by $A P(I: X)$.

Let $1 \leq p<\infty$. Then we say that a function $f \in L_{l o c}^{p}(I$ : $X)$ is Stepanov $p$-bounded, $S^{p}$-bounded shortly, iff:

$$
\|f\|_{S^{p}}:=\sup _{t \in I}\left(\int_{t}^{t+1}\|f(s)\|^{p} d s\right)^{1 / p}<\infty
$$

Endowed by the above norm, the space $L_{S}^{p}(I: X)$ consisting of all $S^{p}$-bounded functions becomes a Banach space. A function $f \in L_{S}^{p}(I: X)$ is said to be Stepanov $p$-almost periodic, Sp-almost periodic shortly, iff the function $\hat{f}: I \rightarrow L^{p}([0,1]: X)$, defined by:

$$
\hat{f}(t)(s):=f(t+s), t \in I, s \in[0,1]
$$


is almost periodic (cf. the monograph by Amerio and Prouse (1971) for more details on the subject). We use the symbol $\operatorname{APS}^{p}(I: X)$ to denote the vector space consisting of all Stepanov p-almost periodic functions. Let us recall that if $f(\cdot)$ is an almost periodic function, then $f(\cdot)$ is also $\mathrm{Sp}$-almost periodic for $1 \leq p<1$. The converse statement is not true, however.

Assume that $f: I \times Y \rightarrow X$. Define $\hat{f}: I \times Y \rightarrow L^{p}([0$, 1]: $X)$ by $\hat{f}(t, y):=f(t+\cdot, y), t>0, y \in Y$.

We need to recall the following definitions (Zhang, 2001; Long and Ding, 2011; Kostić, 2017c):

\section{Definition 2.1}

Suppose that $1 \leq p<1$ :

- A function $f: I \times Y \rightarrow X$ is said to be almost periodic iff $f(\cdot, \cdot)$ is bounded, continuous as well as for every $\epsilon$ $>0$ and every compact $K \subseteq Y$ there exists $l(\epsilon, K)>0$ such that every subinterval $J \subseteq I$ of length $l(\epsilon, K)$ contains a number $\tau$ with the property that $\| f(t+\tau$, $y)-f(t, y) \| \leq \epsilon$ for all $t \in I, y \in K$. The collection of such functions will be denoted by $A P(I \times Y: X)$

- A function $f: I \times Y \rightarrow X$ is called Stepanov $p$-almost periodic, $S^{p}$-almost periodic shortly, iff $\hat{f}: I \times Y \rightarrow$ $L^{p}([0,1]: X)$ is almost periodic. The collection of such functions will be denoted by $\operatorname{APS}^{p}(I \times Y: X)$

It is well known that (Zhang, 2001) if $f \in A P(I \times Y$ : $X)$ and $h \in A P(I: Y)$, then the mapping $t \mapsto f(t, h(t)), t \in$ $I$ belongs to the space $A P(I: X)$.

For more details about Stepanov $p$-almost periodic functions depending on two parameters, we refer the reader to (Kostić, 2017c).

\section{Multivalued Linear Operators}

One of the most important monographs on abstract degenerate differential equations with integer order derivatives was written by Favini and Yagi (1998). In this monograph, the authors obey the multivalued linear operators approach to degenerate differential equations. The main aim of this section is to present a brief overview of definitions and results from this theory (cf. the monograph (Cross, 1998) by Cross for more details on the subject).

A multivalued map (multimap) $A: X \rightarrow P(Y)$ is said to be a Multivalued Linear Operator (MLO) iff the following holds:

- $D(A):=\{x \in X: A x \neq \emptyset\}$ is a linear submanifold of $X$

- $A x+A y \subseteq A(x+y), x, y \in D(A)$ and $\lambda A x \subseteq$ $A(\lambda x), \lambda \in \mathbb{C}, x \in D(A)$. If $X=Y$, then it is said that $A$ is an MLO in $X$
Let us recall that, for every $x, y \in D(A)$ and for every $\lambda, \eta \in \mathbb{C}$ with $|\lambda|+|\eta| \neq 0$, one has $\lambda A x+\eta A y=A(\lambda x+$ $\eta y)$. If $A$ is an MLO, then $A 0$ is a linear sub manifold of $Y$ and $A x=f+A 0$ for any $x \in D(A)$ and $f \in A x$. Put $R(A):=\{A x: x \in D(A)\}$. The set $\mathrm{A}^{-1} 0=\{x \in D(A): 0 \in$ $A x\}$ is called the kernel of $A$ and it is denoted by $N(A)$. The inverse $A^{-1}$ of an MLO is defined by $D\left(A^{-1}\right):=R(A)$ and $A^{-1} y:=\{x \in D(A): y \in A x\}$. It can be simply proved that $A^{-1}$ is an MLO in $X$, as well as that $N\left(A^{-1}\right)=A 0$ and $\left(A^{-1}\right)^{-1}=A$.

Assume that $A, B: X \rightarrow P(Y)$ are two MLOs. Then we define its sum $A+B$ by $D(A+B):=D(A) \cap D(B)$ and $(A+$ $B) x:=A x+B x, x \in D(A+B)$. It is evident that $A+B$ is likewise an MLO.

Assume further that $A: X \rightarrow P(Y)$ and $B: Y \rightarrow P(Z)$ are two MLOs, where $Z$ is likewise a complex Banach space. The product of operators $A$ and $B$ is defined by $D(B A):=\{x \in D(A): D(B) \cap A x \neq \emptyset\}$ and $B A x:=$ $B(D(B) \cap A x)$. We know that $B A: X \rightarrow P(Z)$ is an MLO and $(B A)^{-1}=A^{-1} B^{-1}$.

We say that an MLO operator $A: X \rightarrow P(Y)$ is closed iff for any sequences $\left(x_{n}\right)$ in $D(A)$ and $\left(y_{n}\right)$ in $Y$ such that $y_{n} \in A x_{n}$ for all $x \in \mathbb{N}$ we have that $\lim _{n \rightarrow \infty} x_{n}=x$ and $\lim _{n \rightarrow \infty} y_{n}=y$ imply $x \in D(A)$ and $y \in A x$.

The following auxiliary lemma from integration theory can be found, for example, in (Kostić, 2016).

\section{Lemma 3.1}

Let $\Omega$ be a locally compact, separable metric space and let $\mu$ be a locally finite Borel measure defined on $\Omega$. Assume that $A: X \rightarrow P(Y)$ is a closed MLO. Let $f: \Omega \rightarrow X$ and $g: \Omega \rightarrow Y$ be $\mu$-integrable and let $g(x) \in A f(x), x \in \Omega$. Then $\int_{\Omega} f d \mu \in D(A)$ and $\int_{\Omega} g d \mu \in A \int_{\Omega} f d \mu$.

Let $A$ be an MLO in $X$. Then the resolvent set of $A$, $\rho(A)$ for short, is defined as the union of those complex numbers $\lambda \in \mathbb{C}$ for which:

- $\quad X=R(\lambda-A)$

- $\quad(\lambda-A)^{-1}$ is a single-valued linear continuous operator on $X$

The operator $\lambda \mapsto(\lambda-A)$ is called the resolvent of $A$, $R(\lambda: A) \equiv(\lambda-A)^{-1}(\lambda \in \rho(A))$. The basic properties of resolvents of single-valued linear operators continue to hold in the multivalued linear setting (Favini and Yagi, 1998; Kostić, 2016).

\section{Fractional Powers and Interpolation Spaces of Multivalued Linear Operators}

Let $X$ be a Banach space and let $(-\infty, 0] \subseteq \rho(A)$. Assume that there exist finite numbers $M \geq 1$ and $\beta \in(0$, 1] such that $\|R(\lambda: A)\| \leq M(1+|\lambda|)^{-\beta}, \lambda \leq 0$. Then there 
exist two positive real constants $c>0$ and $M_{1}>0$ such that $\rho(A)$ contains an open region $\Omega=\{\lambda \in \mathbb{C}: \mid \Im \lambda \lambda\} \mid \leq$ $\left.\left(2 M_{1}\right)^{-1}(c-\Re \lambda)^{\beta}, \Re \lambda \leq c\right\}$ of complex plane around the half-line $(-\infty, 0]$, where the following holds: $\|R(\lambda: A)\|=$ $O\left((1+\mid \lambda j)^{-\beta}\right), \lambda \in \Omega$, Designate by $\Gamma^{\prime}$ the upwards oriented curve $\left\{\xi \pm i\left(2 M_{1}\right)^{-1}(c-\xi)^{\beta}:-\infty<\xi \leq c\right\}$. In, Favini and Yagi (1998) define the fractional power:

$$
A^{-\theta}:=\frac{1}{2 \pi i} \int_{\Gamma^{\prime}} \lambda^{-\theta}(\lambda-A)^{-1} d \lambda \in L(X)
$$

For $\theta>1-\beta$. Put $A^{\theta}:=\left(A^{-\theta}\right)^{-1}(\theta>1-\beta)$. Then the semigroup properties $A^{-\theta_{1}} A^{-\theta_{2}}=A^{-\left(\theta_{1}+\theta_{2}\right)} \quad$ and $A^{\theta_{1}} A^{\theta_{2}}=A^{\left(\theta_{1}+\theta_{2}\right)}$ hold for $\theta_{1}, \theta_{2}>1-\beta$ (recall that the fractional power $A^{\theta}$ need not be injective and that $A^{\theta}$ is an MLO for $\theta>1-\beta$ ).

We endow the vector space $D(A)$ by the norm $\|\cdot\|_{[D(A)]}:=\inf _{y \in A} \cdot\|y\|$. Then $\left(D(A),\|\cdot\|_{[D(A)]}\right.$ is a Banach space and the norm $\|\cdot\|_{[D(A)]}$ is equivalent with the following one $\|\cdot\|+\|\cdot\|_{[D(A)]},\left(D\left(A^{\theta}\right),\|\cdot\|_{\left[D\left(A^{\theta}\right)\right]}\right)$ is a Banach space, as well and we have the equivalence of norms $\|\cdot\|_{\left[D\left(A^{\theta}\right)\right]}$ and $\|\cdot\|+\|\cdot\|_{\left[D\left(A^{\theta}\right)\right]}$ for $\theta>1-\beta$.

Assume that $\theta \in(0,1)$. The interpolation space $X_{A}^{\theta}$ is defined by:

$$
X_{A}^{\theta}:=\left\{x \in X: \sup _{\xi>0} \xi^{\theta}\left\|\xi(\xi+A)^{-1} x-x\right\|<\infty\right\}
$$

When equipped with the norm:

$$
\|\cdot\|_{X_{A}^{\theta}}:=\|\cdot\|+\sup _{\xi>0} \xi^{\theta}\left\|\xi(\xi+A)^{-1} \cdot-\cdot\right\|
$$

$X_{A}^{\theta}$ is a Banach space that is continuously embedded in $X$.

For further information concerning interpolation spaces and fractional powers of multivalued linear operators, we refer the reader to (Favaron and Favini, 2011; Favini and Yagi, 1998; Kostić, 2016).

\section{Almost Periodic Solutions of Abstract Semilinear Cauchy Inclusions (1.1) and (1.2)}

A composition theorem for two-parameter Stepanov $p$-almost periodic functions has been clarified in ((Long and Ding, 2011) Theorem 2.2); cf. also (Kostić, 2017c). The following result states that the assertion of (Long and Ding, 2011, Theorem 2.2) continues to hold for the functions defined on the real semi-axis $I=[0, \infty)$, as well, with two different pivot spaces $X$ and $Y$. The proof is very similar to that of afore-mentioned result and therefore omitted.

\section{Theorem 4.1}

Let $I \in \mathbb{R}$ or $I=[0, \infty)$. Suppose that the following conditions hold:

- $f \in \operatorname{APS}^{p}(I \times Y: X)$ with $p>1$; and there exist a number $r \geq \max (p, p / p-1)$ and a function $L_{f} \in L_{S}^{r}(I)$ such that:

$$
\|f(t, x)-f(t, y)\| \leq L_{f}(t)\|x-y\|_{Y}, \quad t \in I, x, y \in Y
$$

- $\quad x \in \operatorname{APS}^{p}(I: Y)$ and there exists a set $E \subseteq I$ with $m(E)$ $=0$ such that $K:=\{x(t): t \in N E\}$ is relatively compact in $Y$; here, $m(\cdot)$ denotes the Lebesgue measure

Then $q:=p r / p+r \in[1, p)$ and $f(\cdot, x(\cdot)) \in A P S^{q}(I: X)$. If we accept the existence of a Lipschitz constant $L>$ 0 such that:

$\|f(t, x)-f(t, y)\| \leq L\|x-y\|_{Y}, \quad t \in I, x, y \in Y$

then we can also consider the case in which $p=1$. Strictly speaking, the following result holds true.

\section{Theorem 4.2}

Let $I \in \mathbb{R}$ or $I=[0, \infty)$. Suppose that the following conditions hold:

- $f \in A P S^{p}(I \times Y: X)$ with $p \geq 1, L>0$ and (4.2) holds

- $\quad x \in A P S^{p}(I: Y)$ and there exists a set $E \subseteq I$ with $m(E)=0$ such that $K=\{x(t): t \in \Lambda E\}$ is relatively compact in $Y$

Then $f(\cdot, x(\cdot)) \in A P S^{p}(I: X)$ :

The following lemma can be proved in exactly the same way as ((Kostić, 2017a), Proposition 2.11).

\section{Lemma 4.1}

Suppose that $1 \leq p<\infty, 1 / p+1 / q=1$ and $(R(t))_{t>0} \subseteq$ $L(X, Y)$ is a strongly continuous operator family satisfying that $M:=\sum_{k=0}^{\infty}\|R(\cdot)\|_{L^{q}[k, k+1]}<\infty$. If $f: \mathbb{R} \rightarrow X$ is $S^{p}$-almost periodic, then the function $G: \mathbb{R} \rightarrow Y$, given by:

$$
G(t):=\int_{-\infty}^{t} R(t-s) f(s) d s, t \in \mathbb{R}
$$

is well-defined and almost periodic.

\section{Remark 4.1}

Let $p>1$ and let $t \mapsto\|R(t)\|, t \in(0,1]$ be an element of the space $L^{q}[0,1]$. Then the inequality $\sum_{k=0}^{\infty}\|R(\cdot)\|_{L^{q}[k, k+1]}<\infty$ holds provided that there exists a 
finite number $\zeta<0$ such that $\|R(t)\|=O\left(t^{\zeta}\right), t \rightarrow+1$ and $\zeta<(1 / p)-1$.

Suppose now that the condition $(\mathrm{P})$ holds and $\beta>\theta$ $>1-\beta$. Then degenerate strongly continuous semigroup $(T(t))_{t>0} \subseteq L(X)$ generated by A satisfies estimate $\|T(t)\| \leq M_{0} e^{-c t} t^{\beta-1} ; t>0$ for some finite constant $M_{0}>0$ (Kostić, 2017a).

Set:

$$
T \gamma, v(t) x:=t^{\gamma v} \int_{0}^{\infty} s^{v} \Phi_{\gamma}(s) T\left(s t^{\gamma}\right) x d s, t>0, x \in X
$$

and, following Wang et al. (2012) (cf. also El-Borai and Debbouche (2009)):

$$
P_{\gamma}(t):=\gamma T_{\gamma, 1}(t) / t^{\gamma}, \quad t>0
$$

In the sequel, by $M>0$ we denote the finite generic constant whose numerical value may change from line to line.

\section{Almost Periodic Solutions of Abstract Semilinear Cauchy Inclusion (1.1)}

Put $Y:=\left[D\left((-A)^{\theta}\right)\right]$ and $\|\cdot\|_{Y:}:=\|\|_{\left[D\left((-A)^{\theta}\right)\right]}$. In (Kostić, $2017 \mathrm{~b}$ ), we have proved that:

$$
\begin{aligned}
& \left(T(t) x, \frac{1}{2 \pi i} \int_{\Gamma}(-\lambda)^{\theta} e^{\lambda t}(\lambda-A)^{-1} x d \lambda\right) \\
& \in(-A)^{\theta}, x \in X, t>0
\end{aligned}
$$

Set:

$$
T_{v}(t) x:=\frac{1}{2 \pi i} \int_{\Gamma}(-\lambda)^{v} e^{\lambda t}(\lambda-A)^{-1} x d \lambda, x \in X, t>0(v>0)
$$

Arguing as in the proof of ((Kostić, 2017b), Lemma 2.6), we get that:

$$
\left\|T_{v}(t)\right\| \leq M e^{-c t} t^{\beta-v-1}, t>0, v>0
$$

Applying the mean value theorem, (A) and the obvious equality $T_{v}^{\prime}(t)=-T_{v+1}(t), t>0, v>0$, we obtain that:

$$
\left\|T_{v}(t+h)-T_{v}(t)\right\| \leq M h e^{-c t} t^{\beta-v-2}, t>0, v>0, h>0
$$

The following notion of a mild solution of (1.1) will be sufficiently good for our purposes:

\section{Definition 4.1}

Let $f: I \times Y \rightarrow X$ and let $Y$ be continuously embedded in $X$. By a mild solution of (1.1), we mean any $Y$-continuous function $u(\cdot)$ such that $u(t)=(\Lambda u)(t), t \in R$, where:

$$
t \mapsto(\Lambda u)(t):=\int_{-\infty}^{t} T(t-s) f(s, u(s)) d s, t \in \mathbb{R}
$$

We continue by stating the following important lemma.

\section{Lemma 4.2}

Let $1<q, q^{\prime}<\infty, 1 / q+1 / q^{\prime}=1$ and $q^{\prime}(\beta-\theta-1)>-1$. Assume that $\hat{f}: I \times Y \rightarrow L^{q}([0,1]: X)$ is bounded continuous and $u \in C(\mathbb{R}: Y)$. Define:

$$
(\Psi u)(t):=\int_{-\infty}^{t} T_{\theta}(t-s) f(s, u(s)) d s, t \in \mathbb{R}
$$

Then $\Psi u \in C_{b}(\mathbb{R}: X)$.

\section{Proof}

Let $\int_{t}^{t+1}\|f(s, y)\|^{q} d s \leq M^{q}$ for all $t \in \mathbb{R}$ and $y \in Y$. We will first prove the right continuity of $(\Psi u)(\cdot)$. For this, fix a number $h \in(0,1]$. Then a straightforward computation involving the Hölder inequality shows that:

$$
\begin{aligned}
& \|(\Psi u)(t+h)-(\Psi u)(t)\| \\
& \leq \int_{-\infty}^{t}\left\|T_{\theta}(T+h-s)-T_{\theta}(t-s)\right\|\|f(s, u(s))\| d s \\
& +\int_{-\infty}^{t+h}\left\|T_{\theta}(T+h-s)\right\|\|f(s, u(s))\| d s \\
& =\int_{0}^{\infty}\left\|T_{\theta}(s+h)-T_{\theta}(s)\right\|\|f(t-s, u(t-s))\| d s \\
& +\int_{0}^{h}\left\|T_{\theta}(s+h)\right\|\|f(t-s, u(t-s))\| d s \\
& \leq \int_{0}^{1}\left\|T_{\theta}(s+h)-T_{\theta}(s)\right\|\|f(t-s, u(t-s))\| d s \\
& +\sum_{k=1}^{\infty} \int_{k}^{k+1}\left\|T_{\theta}(s+h)-T_{\theta}(s)\right\|\|f(t-s, u(t-s))\| d s \\
& +\int_{0}^{h}\left\|T_{\theta}(s+h)\right\|\|f(t-s, u(t-s))\| d s \\
& \leq M\left(\int_{0}^{1}\left\|T_{\theta}(s+h)-T_{\theta}(s)\right\|^{q^{\prime}} d s\right)^{1 / q^{\prime}} \\
& +M \sum_{k=1}^{\infty} \int_{0}^{k+1}\left\|T_{\theta}(\cdot+h)-T_{\theta}(\cdot)\right\|_{L^{q^{\prime}}[k, k+1]} \\
& +M\left(\int_{0}^{h} \| T_{\theta}(s+h)^{q^{\prime}} d s\right)^{1 / q^{\prime}}, t \in \mathbb{R}
\end{aligned}
$$

which clearly implies that:

$$
\begin{aligned}
& \|(\Psi u)(t+h)-(\Psi u)(t)\| \\
& \leq M\left(\int_{0}^{1}\left\|T_{\theta}(s+h)-T_{\theta}(s)\right\|^{q^{\prime}} d s\right)^{1 / q^{\prime}} \\
& +M \sum_{k=1}^{\infty} \int_{0}^{k+1}\left\|T_{\theta}(\cdot+h)-T_{\theta}(\cdot)\right\|_{L^{\infty}[k, k+1]} \\
& +M\left(\int_{0}^{2 h} \| T_{\theta}(s)^{q^{\prime}} d s\right)^{1 / q^{\prime}}
\end{aligned}
$$


for any $t \in \mathbb{R}$. It is clear that the third addend in the last expression tends to zero as $h$ tends to zero because of (A) and $q^{\prime}(\beta-\theta-1)>-1$, which gives that $\left\|T_{\theta}(\cdot)\right\|^{q^{\prime}} \in L^{1}[0,1]$. For the second addend, we can use the estimate:

$$
\sum_{k=1}^{\infty} \int_{k}^{k+1}\left\|T_{\theta}(\cdot+h)-T_{\theta}(\cdot)\right\|_{L^{\infty}[k, k+1]} \leq M h \sum_{k=1}^{\infty} e^{-c k}
$$

which simply follows from (B). For the first addend, we can employ the dominated convergence theorem and (A); summa summarum, we have proved the right continuity of $(\Psi u)(\cdot)$. The left continuity of $(\Psi u)(\cdot)$ can be proved similarly and we only yet need to show the boundedness of $(\Psi u)(\cdot)$. But, this is a consequence of the following calculus obtained with the help of Hölder inequality:

$$
\begin{aligned}
& \|(\Psi u)(t)\| \\
& \leq \int_{0}^{\infty}\left\|T_{\theta}(s)\right\|\|f(t-s, u(t-s))\| d s \\
& \leq M \sum_{k=0}^{\infty}\left(\int_{k}^{k+1}\left\|T_{\theta}(s)\right\|^{q^{\prime}} d s\right)^{1 / q^{\prime}}
\end{aligned}
$$

which is valid for any $t \in \mathbb{R}$ and the estimate (A).

Define, for every $X$-valued bounded continuous function $u(\cdot)$ :

$$
(\Phi u)(t):=\int_{-\infty}^{t} T_{\theta}(t-s) f\left(s,(-A)^{-\theta} u(s)\right) d s, t \in \mathbb{R}
$$

Since $(-A)^{-\theta} \in L\left(X,\left[D\left((-A)^{\theta}\right)\right]\right)$, we know from Lemma 4.2 that $\Phi: C_{b}(\mathbb{R}: X) \rightarrow C_{b}(\mathbb{R}: X)$ is well-defined as long as $f(\cdot, \cdot)$ satisfies the conditions from Lemma 4.2.

Let $L_{f}(\cdot)$ be a locally bounded non-negative function and let $\mathrm{M}$ denote the constant from (A), with $v=\theta$. Set, for every $n \in \mathbb{N}$ :

$$
\begin{aligned}
& M_{n}:=M^{n} \sup _{t \in \mathbb{R}} \int_{-\infty}^{t} \int_{-\infty}^{x_{n}} \cdots \int_{-\infty}^{x_{2}} e^{-c\left(t-x_{n}\right)}\left(t-x_{n}\right)^{\beta-\theta-1} \\
& \times \prod_{i=2}^{n} e^{-c\left(x_{i}-x_{i-1}\right)}\left(x_{i}-x_{i-1}\right)^{\beta-\theta-1} \prod_{i=1}^{n} L_{f}\left(x_{i}\right) d x_{1} d x_{2} \cdots d x_{n}
\end{aligned}
$$

Since the norm of mapping $(-A)^{-\theta} \in L\left(X,\left[D\left((-A)^{\theta}\right)\right]\right)$ is less or equal than 1 , a simple calculation yields that:

$$
\left\|\left(\Phi^{n} u\right)-\left(\Phi^{n} v\right)\right\|_{\infty} \leq M_{n}\|u-v\|_{\infty}, u, v \in C_{b}(\mathbb{R}: X), n, \mathbb{N}
$$

Now we are ready to formulate the following result.

\section{Theorem 4.3}

Suppose that $(\mathrm{P})$ holds, $\beta>\theta>1-\beta$ and the following conditions hold: i. $f \in A P S^{p}(R \times Y: X)$ with $p>1$ and there exist a number $r \geq \max (p, p / p-1)$ as well as a locally bounded non-negative function $L_{f} \in L_{S}^{r}(\mathbb{R})$ such that $r>p / p-1$ and (4.1) holds.

Set $q:=p r / p+r$ and $q^{\prime}:=\frac{p r}{p r-p-r}$.

Assume also that:

ii. $q^{\prime}(\beta-\theta-1)>-1$

iii. $M_{n}<1$ for some $n \in \mathbb{N}$

Then there exists an almost periodic mild solution of inclusion (1.1). The uniqueness of mild solutions holds provided that, in addition to (i-iii), $A$ is singlevalued and:

iv. $\sum_{n=1}^{\infty} M_{n}<\infty$ or $M_{1}<1$

\section{Proof}

Let us recall that the range of a $Y$-valued almost periodic function is relatively compact in $Y$ by ((Zhang, 2001), Theorem 2.4). Clearly, $u \in A P(R: X)$ implies $(-A)^{\theta} u$ $\in A P(\mathbb{R}: Y)$; after that, using the condition (i) and Theorem 4.1, we get that $f\left(\cdot,(-A)^{\theta} u(\cdot)\right) \in A P S^{q}(\mathbb{R}: Y)$. Due to the assumption (ii), the inequality (A) and Lemma 4.1 (see also Remark 4.1), we get that the mapping $\Phi_{\mid A P(\mathbb{R}: Y)}: A P(\mathbb{R}: X) \rightarrow A P(\mathbb{R}: X)$ is well-defined. Since (4.6) and (iii) hold, we can apply a well known extension of the Banach contraction principle in order to see that the mapping $\Phi_{\mid A P(\mathbb{R}: Y)}: A P(\mathbb{R}: X) \rightarrow A P(\mathbb{R}: X)$ has a fixed point $\varphi(\cdot)$. Observing that $q^{\prime}(\beta-1)>-1$ by (ii), we can employ Lemma 4.1 again to conclude that the mapping $t \mapsto \int_{-\infty}^{t} T(t-s) f\left(s,(-A)^{-\theta} \varphi(s)\right) d s, t \in \mathbb{R}$ is well-defined. Due to Lemma 3.1 and (4.4), we obtain that:

$$
\begin{aligned}
& \varphi(t)=\int_{-\infty}^{t} T_{\theta}(t-s) f\left(s,(-A)^{-\theta} \varphi(s)\right) d s \\
& \in(-A)^{\theta} \int_{-\infty}^{t} T(t-s) f\left(s,(-A)^{-\theta} \varphi(s)\right) d s, t \in \mathbb{R}
\end{aligned}
$$

This implies:

$$
(-A)^{-\theta} \varphi(t)=\int_{-\infty}^{t} T(t-s) f\left(s,(-A)^{-\theta} \varphi(s)\right) d s, t \in \mathbb{R}
$$

Taking into account that:

$$
\left\|(-A)^{-\theta} \varphi(t)-(-A)^{-\theta} \varphi(s)\right\|_{Y} \leq\|\varphi(t)-\varphi(s)\|, t, s \in \mathbb{R}
$$

the mapping $(-A)^{-\theta} \varphi(\cdot)$ is $Y$-continuous so that, actually, $(-A)^{-\theta} \varphi(\cdot)$ is an almost periodic mild solution of (1.1) (by 
almost periodicity, we mean the $X$-almost periodicity). To complete the proof of theorem, we need to show the uniqueness of mild solutions in the case that $A=A$ is single-valued and (iv) holds. Then the fractional powers constructed in Subsection 3.1 coincide with those constructed in (Periago and Straub, 2002), so that the fractional powers $(-A)^{ \pm \theta}$ are injective. Assume that $u(\cdot)$ is a mild solution of (1.1); hence, $u(t)=\int_{-\infty}^{t} T(t-$ $s) f(s, u(s)) d s, t \in \mathbb{R}$. Then:

$$
\begin{aligned}
& u(t)=\int_{-\infty}^{t} T(t-s) f(s, u(s)) d s \\
& =\int_{-\infty}^{t}(-A)^{-\theta} T_{\theta}(t-s) f(s, u(s)) d s \\
& =(-A)^{-\theta} \int_{-\infty}^{t} T_{\theta}(t-s) f(s, u(s)) d s:=(-A)^{-\theta} \zeta(t), t \in \mathbb{R}
\end{aligned}
$$

observe here that the mapping $\zeta(\cdot)$ is well-defined, $X$ continuous and $X$-bounded by Lemma 4.2 because $1 / q+$ $1 / q^{\prime}=1$ and $q \in(1, p)$. This implies:

$$
\begin{aligned}
& (-A)^{-\theta} \zeta(t)=u(t)=(-A)^{-\theta} \int_{-\infty}^{t} T_{\theta}(t-s) f(s, u(s)) d s \\
& =(-A)^{-\theta} \int_{-\infty}^{t} T_{\theta}(t-s) f\left(s,(-A)^{-\theta} \zeta(s)\right) d s \quad t \in \mathbb{R}
\end{aligned}
$$

By the injectivity of power $(-A)^{-\theta}$, we get that $\zeta(\cdot)$ is a fixed point of mapping $\Phi: C_{b}(\mathbb{R}: X) \rightarrow C_{b}(\mathbb{R}: X)$. On the other hand, the validity of condition (iv) enables one to apply Weissinger's fixed point theorem ((Diethelm, 2010), Theorem D.7) or the classical Banach contraction principle, showing that there exists a unique fixed point of $\Phi(\cdot)$ in $C_{b}(\mathbb{R}: X)$. Hence, $\zeta(\cdot)$ is uniquely determined and, because of that, $u(\cdot)$ is uniquely determined, as well.

Assuming the Lipschitz condition (4.2) in place of (4.1), we can deduce the following result.

\section{Theorem 4.4}

Suppose that (P) holds, $\beta>\theta>1-\beta$ and the following conditions hold:

i. $f \in A P S^{p}(\mathbb{R} \times Y: X)$ with $p>1$ and there exists a constant $L>0$ such that (4.2) holds

ii. $\frac{p}{p-1}(\beta-\theta-1)>-1$

iii. $M_{n}<1$ for some $n \in \mathbb{N}$

Then there exists an almost periodic mild solution of inclusion (1.1). The uniqueness of mild solutions holds provided that, in addition to (i-iii), $A$ is single-valued:

iv. $\sum_{n=1}^{\infty} M_{n}<\infty$ or $M_{1}<1$
Before proceeding further, it would be worthwhile to mention that a direct computation shows that, in the concrete situation of Theorem 4.4, one has:

$$
M_{1} \leq M L \Gamma(\beta-\theta) c^{\theta-\beta}
$$

where, $M$ denotes the constant from (A), with $v=\theta$.

\section{Almost Periodic Solutions of Abstract Semilinear Cauchy Inclusion (1.2)}

In this subsection, we examine the existence and uniqueness of almost periodic solutions of abstract fractional inclusion (1.2). We start with the observation that $\mathrm{Mu}$ et al. (2017) have investigated various types of (asymptotically) quasi-periodic and quasi-automorphic solutions of (1.2) provided that the operator $A$ is singlevalued and generates an exponentially decaying strongly continuous semigroup; cf. also (El-Borai and Debbouche, 2009). By performing the Fourier transform in (Mu et al., 2017) Lemma 6], the authors have proposed the following notion of mild solution of (1.2): A continuous function $u: \mathbb{R} \rightarrow X$ is said to be a mild solution of (1.2) iff:

$$
u(t)=\int_{-\infty}^{t}(t-s)^{\gamma-1} P \gamma(t-s) f(s, u(s)) d s, t \in \mathbb{R}
$$

cf. (4.3). As already mentioned in (Kostić, 2017b), the method followed in the proof of ((Mu et al., 2017), Lemma 6 ) is completely meaningful in the case that $A$ is an MLO satisfying the condition (P). In our approach, we need to have two different pivot spaces for a reasonable definition of a mild solution of (1.2); see also Definition 4.1.

\section{Definition 4.2}

Let $f: I \times Y \rightarrow X$ and let $Y$ be continuously embedded in $X$. By a mild solution of (1.2), we mean any $Y$-continuous function $u(\cdot)$ such that $u(t)=(\Lambda \gamma)(t), t \in \mathbb{R}$, where:

$$
t \mapsto\left(\Lambda_{\gamma} u\right)(t):=\int_{-\infty}^{t}(t-s)^{\gamma-1} P_{\gamma}(t-s) f(s, u(s)) d s, t \in \mathbb{R}
$$

Set:

$$
R_{\gamma}(t):=t^{\gamma-1} P_{\gamma}(t), t>0
$$

and:

$$
R_{\gamma}^{\theta}(t):=\gamma t^{\gamma-1} \int_{0}^{\infty} s \Phi_{\gamma}(s) T_{\theta}\left(s t^{\gamma}\right) x d s, t>0, x \in X
$$

We need to prove a few auxiliary lemmae.

\section{Lemma 4.3}

There exists a finite constant $M_{\gamma}^{\theta}>0$ such that: 


$$
\left\|R_{\gamma}^{\theta}(t)\right\| \leq M_{\gamma}^{\theta} t^{\gamma(\beta-\theta)-1}, t \in(0,1] \text { and }\left\|R_{\gamma}^{\theta}(t)\right\| \leq M_{\gamma}^{\theta} t^{-1-\gamma}, t \geq 1
$$

Furthermore:

$$
R_{\gamma}^{\theta}(t) x \in(-A)^{\theta} R_{\gamma}(t) x, t>0, x \in X
$$

\section{Proof}

By introduced definitions, we have:

$$
(-A)^{\theta} R_{\gamma}(t) x=\gamma t^{\gamma-1}(-A)^{\theta}\left[\int_{0}^{\infty} s \Phi_{\gamma}(s) T\left(s t^{\gamma}\right) x d s\right], t>0, x \in X
$$

Since the power $(-A)^{\theta}$ is closed, we can apply Lemma 3.1 and (4.4) to see that (4.8) holds good. On the other hand, a straightforward computation yields:

$$
\left\|R_{\gamma}^{\theta}(t)\right\| \leq M_{\gamma}^{\theta} t^{\gamma(\beta-\theta)-1} \int_{0}^{\infty} e^{-c s t^{\gamma}} \Phi_{\gamma}(s) s^{\beta-\theta} d s
$$

for any $t>0$. Since $\Phi_{\gamma}(s) \sim(\Gamma(1-\gamma))^{-1}, s \rightarrow 0+$, a Tauberian type theorem ((Arendt et al., 2001), Proposition 4.1 .4 ; b) immediately implies the second estimate in (4.7). The first estimate in (4.7) is clear, since $e^{-c s t^{\gamma}} \leq 1$ for $t, s>0$ and the integral $\int_{0}^{\infty} \Phi_{\gamma}(s) s^{\beta-\theta} d s$ converges.

\section{Lemma 4.4}

There exists a finite constant $M_{\gamma, \theta}>0$ such that:

$$
\left\|R_{\gamma}^{\theta}(t+h)-R_{\gamma}^{\theta}(t)\right\| \leq M_{\gamma, \theta} h t^{-2-\gamma}, t \geq 1, h>0
$$

\section{Proof}

By the mean value theorem, it suffices to show that $\left\|(d / d t) R_{\gamma}^{\theta}(t)\right\| O\left(t^{-2-\gamma}\right), t \geq 1$. Towards this end, observe that:

$$
\begin{aligned}
& (d / d t) R_{\gamma}^{\theta}(t) x=\gamma(\gamma-1) t^{\gamma-2} \int_{0}^{\infty} s \Phi_{\gamma}(s) T_{\theta}\left(s t^{\gamma}\right) x d s \\
& -\gamma^{2} t^{2 \gamma-2} \int_{0}^{\infty} s \Phi_{\gamma}(s) T_{\theta+1}\left(s t^{\gamma}\right) x d s
\end{aligned}
$$

for any $t \geq 1$ and $x \in X$. Therefore, making use of the estimate (A) we get:

$$
\begin{aligned}
& \left\|(d / d t) R_{\gamma}^{\theta}(t)\right\| \leq \gamma(1-\gamma) t^{\gamma-2} \int_{0}^{\infty} s \Phi_{\gamma}(s) e^{-x s t^{\gamma}}\left(s t^{\gamma}\right)^{\beta-\theta-1} d s \\
& +\gamma^{2} t^{2 \gamma-2} \int_{0}^{\infty} s \Phi_{\gamma}(s) e-c s t^{\gamma}\left(s t^{\gamma}\right)^{\beta-\theta-2} d s
\end{aligned}
$$

for any $t \geq 1$. Now the final conclusion follows from an application of ((Arendt et al., 2001), Proposition 4.1.4; b).

\section{Lemma 4.5}

Let $1<q, q^{\prime}<1,1 / q+1 / q^{\prime}=1$ and $q^{\prime}(\gamma(\beta-\theta)-1)>-1$. Assume that $\hat{f}: I \times Y \rightarrow L^{q}([0,1]: X)$ is bounded continuous and $u \in C(\mathbb{R}: Y)$. Define:

$$
\left(\Psi_{\gamma}^{\theta} u\right)(t):=\int_{-\infty}^{t} R_{\gamma}^{\theta}(t-s) f(s, u(s)) d s, t \in \mathbb{R}
$$

Then $\Psi_{\gamma}^{\theta} u \in C^{b}(\mathbb{R}: X)$.

\section{Proof}

The proof of lemma is almost the same as that of Lemma 4.2 and the only thing that should be explained in more detail is the fact that the second addend in the last inequality of (4.5) tends to zero as $h$ tends to zero. This is a consequence of the following computation involving the mean value theorem and the estimate (4.7):

$$
\begin{aligned}
& \sum_{k=1}^{\infty} \int_{k}^{k+1}\left\|T_{\theta}(\cdot+h)-T_{\theta}(\cdot)\right\|_{L q^{\prime}[k, k+1]} \\
& \leq h M_{\gamma, \theta} \sum_{k=1}^{\infty}\left(\int_{k}^{k+1} s^{q^{\prime}(-2-\gamma)} d s\right)^{1 / q^{\prime}} \\
& =h M_{\gamma, \theta}\left|q^{\prime}(-2-\gamma)+1\right|^{-1} \sum_{k=1}^{\infty}\left|(k+1)^{q^{\prime}(-2-\gamma)+1}-k^{q^{\prime}(-2-\gamma)+1}\right|^{1 / q^{\prime}} \\
& \leq h M_{\gamma, \theta} \sum_{k=1}^{\infty}\left(k^{q^{\prime}(-2-\gamma)}\right)^{1 / q^{\prime}}=h M_{\gamma, \theta} \sum_{k=1}^{\infty} k^{-2-\gamma}
\end{aligned}
$$

Suppose that (4.1) holds for a.e. $t>0$, with locally bounded non-negative function $L_{f}(\cdot)$. Set, for every $n \in \mathbb{N}$ :

$$
\begin{aligned}
& B_{n}:=\sup _{t \geq 0} \int_{-\infty}^{t} \int_{-\infty}^{x_{n}} \cdots \int_{-\infty}^{x_{2}}\left\|R_{\gamma}^{\theta}\left(t-x_{n}\right)\right\| \\
& \times \prod_{i=2}^{n}\left\|R_{\gamma}^{\theta}\left(x_{i}-x_{i-1}\right)\right\| \prod_{i=1}^{n} L_{f}\left(x_{i}\right) d x_{1} d x_{2} \cdots d x_{n}
\end{aligned}
$$

Let $f(\cdot, \cdot)$ satisfy the conditions from Lemma 4.5. Define, for every $X$-valued bounded continuous function $u(\cdot)$ :

$$
\left(\Phi_{\gamma}^{\theta} u\right)(t):=\int_{-\infty}^{t} R_{\gamma}^{\theta}(t-s) f\left(s,(-A)^{-\theta} u(s)\right) d s, t \in \mathbb{R}
$$

Then we know from Lemma 4.5 that $\Phi_{\gamma}^{\theta}: C_{b}(\mathbb{R}: X) \rightarrow C_{b}(\mathbb{R}: X)$ is well-defined. A simple calculation shows that:

$$
\begin{aligned}
& \left\|\left(\Phi_{\gamma}^{\theta}\right)^{n} u-\left(\Phi_{\gamma}^{\theta}\right)^{n} v\right\|_{\infty} \\
& \leq B_{n}\|u-v\|_{\infty}, u, v \in C_{b}(\mathbb{R}: X), n \in \mathbb{N}
\end{aligned}
$$

Keeping in mind (4.9) and the last three lemmae, we can repeat almost verbatim the proof of Theorem 4.3 
(with the mapping $\Phi(\cdot)$ as well as the operator families $T(\cdot)$ and $T_{\theta}(\cdot)$ replaced by the mapping $\Phi_{\gamma}^{\theta}(\cdot)$ as well as the operator families $R_{\gamma}(\cdot)$ and $R_{\gamma}^{\theta}(\cdot)$, respectively) in order to see that the following results hold true.

\section{Theorem 4.5}

Suppose that (P) holds, $\beta>\theta>1-\beta$ and the following conditions hold:

i. $f \in A P S^{p}(\mathbb{R} \times Y: X)$ with $p>1$; and there exist a number $r>\max (p, p / p-1)$ as well as a locally bounded non-negative function $L_{f} \in L_{S}^{r}(\mathbb{R})$ such that $r>p / p-1$ and (4.1) holds.

$$
\text { Set } q:=p r / p+r \text { and } q^{\prime}:=\frac{p r}{p r-p-r}
$$

Assume also that:

ii. $\left.q^{\prime}(\alpha \beta-\theta)-1\right)>-1$

iii. $B_{n}<1$ for some $n \in \mathbb{N}$

Then there exists an almost periodic mild solution of inclusion (1.1). The uniqueness of mild solutions holds provided that, in addition to (i-iii), $A$ is singlevalued and:

iv. $\quad \sum_{n=1}^{\infty} B_{n}<\infty$ or $B_{1}<1$

\section{Theorem 4.6}

Suppose that $(\mathrm{P})$ holds, $\beta>\theta>1-\beta$ and the following conditions hold:

i. $f \in A P S^{p}(\mathbb{R} \times Y: X)$ with $p>1$ and there exists a constant $L>0$ such that (4.2) holds.

ii. $\left.\frac{p}{p-1}(\alpha \beta-\theta)-1\right)>-1$

iii. $B_{n}<1$ for some $n \in \mathbb{N}$

Then there exists an almost periodic mild solution of inclusion (1.1). The uniqueness of mild solutions holds provided that, in addition to (i-iii), A is single-valued and:

iv. $\quad \sum_{n=1}^{\infty} B_{n}<\infty$ or $B_{1}<1$

Direct computation of coefficient $B_{1}$, carried out with the help of estimate (4.7), shows that:

$$
B_{1} \leq M_{\gamma}^{\theta} L\left[\frac{1}{\gamma(\beta-\theta)}+\frac{1}{\gamma}\right]
$$

We close the paper by providing the following illustrative example.

\section{Example 4.1}

It is clear that our results can be applied in the analysis of existence and uniqueness of almost periodic solutions of non-degenerate semilinear problems with elliptic operators of order $2 m$ in the Hölder space $X=C^{\alpha}(\bar{\Omega})$, where $\Omega$ is a bounded domain in $\mathbb{R}^{n}$ with boundary of class $C^{A m}$, when we have the precise value of the exponent $\beta=1-\frac{\alpha}{2 m}$; for more details, see von Wahl (1972). Concerning degenerate differential inclusions, possible applications can be made in the analysis of existence and uniqueness of almost periodic solutions of the following Poisson semilinear heat equation in the Lebesgue space $X=L^{p}(\Omega)$ :

$$
\begin{aligned}
& \frac{\partial}{\partial t}[m(x) v(t, x)]=(\Delta-b) v(t, x) \\
& +f(t, m(x) v(t, x)), t \in \mathbb{R}, x \in \Omega
\end{aligned}
$$

where, $\Omega$ is a bounded domain in $\mathbb{R}^{n}, b>0, m(x) \geq 0$ a.e. $x \in \Omega, m \in L^{\infty}(\Omega), 1<p<\infty$ and the operator $\Delta$ - $b$ acts on $X$ with the Dirichlet boundary conditions. It is clear that Theorem 4.3 and Theorem 4.4 require the condition $\beta>1 / 2$, which is slightly restrictive in degenerate case (in a great deal of examples from [(Favini and Yagi, 1998), Chapter III], the condition (P) holds with $\beta=1 / 2$ ), these theorems can be applied by imposing the additional condition [(Favini and Yagi, 1998), (3.42)] on the function $m(x)$, which leads us to the better exponent $\beta=1 /(2-\rho)$ in $(\mathrm{P})$, with $0<\rho$ $\leq 1$. Under the same conditions, we can apply Theorem 4.5 and Theorem 4.6 in the analysis of existence and uniqueness of almost periodic solutions of the following fractional Poisson semilinear heat equation in the Lebesgue space $L^{p}(\Omega)$ :

$$
\begin{aligned}
& D_{t,+}^{\gamma}[m(x) v(t, x)]=(\Delta-b) v(t, x) \\
& +f(t, m(x) v(t, x)), t \in \mathbb{R}, x \in \Omega
\end{aligned}
$$

Finally, we would like to propose the problem of transferring our main results to the case where the pivot space $\mathrm{Y}$ is no longer $\left[D\left((-A)^{\theta}\right)\right]$ but the interpolation space $X_{A}^{\theta}$.

\section{Conclusion}

In this study, we have investigated the existence of a unique almost periodic solution for a class of abstract fractional relaxation inclusions with Weyl-Liouville derivatives. The analysis of existence and uniqueness of quasi-periodic and quasiautomorphic solutions to 
abstract degenerate Volterra integro-differential equations is not well explored in the existing literature. We deeply believe that a bigger group of mathematicians will continue their scientific work within this field of functional analysis in the near future.

\section{Acknowledgment}

The author truly appreciates the generous support of my family, colleagues and closest friends, whose advices played a mayor role for the beginning of this research study.

\section{Ethics}

The author hereby certifies that he has no financial or non-financial interest in regard to the subject matter discussed in this manuscript.

\section{References}

Amerio, M. and G. Prouse, 1971. Almost-Periodic Functions and Functional Equations. 1st Edn., Van Nostrand-Reinhold, New York, ISBN-10: 0442202954, pp: 184.

Arendt, W., C.J.K. Batty, M. Hieber, F. Neubrander, 2001. Vector-valued Laplace Transforms and Cauchy Problems. 1st Edn., Birkhäuser, Basel, ISBN-10: 3764365498, pp: 523.

Bahaj, M. and O. Sidki, 2002. Almost periodic solutions of semilinear equations with analytic semigroups in Banach spaces, Electronic J. Differ. Eq., 98: 1-11.

Bazhlekova, E., 2001. Fractional evolution equations in banach spaces. PhD Thesis, Eindhoven University of Technology, Eindhoven.

Cross, R., 1998. Multivalued Linear Operators. 1st Edn., CRC Press, ISBN-10: 0824702190, pp: 352.

Diagana, T., 2013. Almost Automorphic Type and Almost Periodic Type Functions in Abstract Spaces. 1st Edn., Springer-Verlag, New York, ISBN-10: 3319008498, pp: 303.

Diagana, T., C.M. Mahop and G.M. N'Guérékata, 2006. Pseudo-almost-periodic solutions to some semilinear differential equations. Math. Comput. Model., 43: 89-96. DOI: $10.1016 /$ j.mcm.2005.04.013

Diethelm, K., 2010. The Analysis of Fractional Differential Equations. 1st Edn., Springer-Verlag, Berlin, ISBN-10: 3642145736,pp: 247.

El-Borai, M.M. and A. Debbouche, 2009. Almost periodic solutions of some nonlinear fractional differential equations. Int. J. Contemp. Math. Sci., 4: 1373-1387.
Favaron, A. and A. Favini, 2011. Fractional powers and interpolation theory for multivalued linear operators and applications to degenerate differential equations. Tsukuba J. Math., 35: 259-323. DOI: $10.21099 / \mathrm{tkbjm} / 1331658708$

Favini, A. and A. Yagi, 1998. Degenerate Differential Equations in Banach Spaces. 1st Edn., CRC Press, New York, ISBN-10: 0824716779, pp: 336.

N'Guérékata, G.M., 2001. Almost Automorphic and Almost Periodic Functions in Abstract Spaces. 1st Edn., Kluwer Acad. Publ., New York, ISBN-10: 0306466864, pp: 138.

Hino, Y., T. Naito, N.V. Minh and J.S. Shin, 2002. Almost Periodic Solutions of Differential Equations in Banach Spaces. 1st Edn., CRC Press, ISBN-10: 0415272661, pp: 264.

Kostić, M., 2011. Generalized Semigroups and Cosine Functions. 1st Edn., Mathematical Institute SANU, Beograd, ISBN-10: 8680593451, pp: 352.

Kostic, M., 2015. Abstract Volterra Integro-Differential Equations. 1st Edn., CRC Press, Boca Raton, ISBN-10: 148225431X, pp: 484.

Kostić, M., 2016. Abstract Degenerate Volterra IntegroDifferential Equations: Linear Theory and Applications. 1st Edn., Book Manuscript.

Kostić, M., 2017a. Abstract Volterra integro-differential equations: Generalized almost periodicity and asymptotical almost periodicity of solutions. Electronic J. Differ. Equ., submitted.

Kostić, M., 2017b. Asymptotically almost periodic solutions of fractional relaxation inclusions with Caputo derivatives. Publ. Inst. Math., Nouv. Ser.

Kostić, M., 2017c. The existence and uniqueness of almost periodic and asymptotically almost periodic solutions of semilinear Cauchy inclusions. Hacet. J. Math. Stat., submitted.

Levitan, M. and V.V. Zhikov, 1982. Almost Periodic Functions and Differential Equations. 1st Edn., CUP Archive, London, ISBN-10: 0521244072, pp: 211.

Long, W. and S.H. Ding, 2011. Composition theorems of Stepanov almost periodic functions and Stepanovlike pseudo-almost periodic functions, Adv. Difference Eq., 2011: 654695-654695. DOI: $10.1155 / 2011 / 654695$

Mu, J., Y. Zhoa and L. Peng, 2017. Periodic solutions and S-asymptotically periodic solutions to fractional evolution equations. Discrete Dyn. Nat. Soc., 2017: 1364532-1364543. DOI: $10.1155 / 2017 / 1364532$

Pazy, A., 1983. Semigroups of Linear Operators and Applications to Partial Differential Equations. 1st Edn., Springer-Verlag, Berlin, ISBN-10: 3540908455, pp: 279. 
Periago, F. and B. Straub, 2002. A functional calculus for almost sectorial operators and applications to abstract evolution equations. J. Evolut.. Eq., 2: 41-68. DOI: 10.1007/s00028-002-8079-9

Prüss, J., 1993. Evolutionary Integral Equations and Applications. 1st Edn., Birkhèauser Verlag, Basel, ISBN-10: 0817628762, pp: 366.

Samko, S.G., A.A. Kilbas and O.I. Marichev, 1993. Fractional Integrals and Derivatives: Theory and Applications. 1st Edn., Gordon and Breach, New York, ISBN-10: 2881248640, pp: 1016.

von Wahl, W., 1972. Gebrochene Potenzen eines elliptischen Operators und parabolische Differentialgleichungen in Raumen hölderstetiger Funktionen. Nachr. Akad. Wiss. Gottingen Math. Phys., 11: 231-258.
Wang, R.N., D.H. Chen and T.J. Xiao, 2012. Abstract fractional Cauchy problems with almost sectorial operators. J. Differ. Eq., 252: 202-235.

DOI: $10.1016 /$ j.jde.2011.08.048

Zhang, C., 2001. Ergodicity and asymptotically almost periodic solutions of some differential equations. IJMMS 25: 787-800.

DOI: $10.1155 / \mathrm{S} 016117120100429 \mathrm{X}$ 